\title{
Prediction of Treatment Outcome From Relationship Variables in Child and Adolescent Therapy: A Meta-Analytic Review
}

\author{
Stephen R. Shirk and Marc Karver \\ University of Denver
}

\begin{abstract}
Results from 23 studies examining associations between therapeutic relationship variables and treatment outcomes in child and adolescent therapy were reviewed with meta-analytic procedures. Results indicated that the overall strength of the relationship-outcome associations was modest and quite similar to results obtained with adults. This modest association was moderated by 1 substantive factor, type of patient problem, and 5 methodological factors, timing and source of relationship measurement, type and source of outcome, and shared versus cross-source measurement of relationship and outcome variables. Type, mode, structure, and context of treatment did not moderate associations between relationship variables and outcomes. Findings indicated that the association between the therapeutic relationship and treatment outcome was consistent across developmental levels and across diverse types and contexts of child and adolescent therapy. Recommendations for future process research on the therapeutic relationship in child psychotherapy are offered.
\end{abstract}

Two meta-analytic reviews of alliance-outcome relations have revealed modest, but consistent, predictive relationships across types of treatment and types of disorders (Horvath \& Symonds, 1991; Martin, Graske, \& Davis, 2000). However, a limitation of these reviews has been the exclusion of child and adolescent treatment studies. Admittedly, child psychotherapy process research has lagged behind its adult counterpart (Kazdin, Bass, Ayers, \& Rodgers, 1990; Russell \& Shirk, 1998), but there is growing interest in process predictors of treatment outcome with children and adolescents (Kazdin \& Kendall, 1998; Weisz, Huey, \& Weersing, 1998). Further, the therapeutic relationship has been posited to play a critical role in child treatment (Goldfried, 1998; Shirk \& Saiz, 1992) and is one of the few process variables that has received attention in the child therapy outcome literature (Russell \& Shirk, 1998). The primary aim of this meta-analysis is to examine the association between the therapeutic relationship and treatment outcome in child and adolescent therapy. ${ }^{1}$

For many years, the therapeutic relationship has been viewed as a pivotal change mechanism in child psychotherapy, especially in psychodynamic and experiential traditions (Axline, 1947; Freud, 1946). More recently, the therapeutic relationship has been accorded a more prominent role in behavioral and cognitivebehavioral therapy (CBT) with children. For example, Kendall (1991) noted that a positive therapeutic relationship is essential for CBT with youth and has found that the therapeutic relationship is viewed as highly important to children who have completed a course of CBT (Southam-Gerow \& Kendall, 1996).

Despite long-standing clinical and emerging empirical interest in the therapeutic relationship, child process research has yet to

Stephen R. Shirk and Marc Karver, Department of Psychology, University of Denver.

Marc Karver is now at the Department of Psychology, University of South Florida.

Correspondence concerning this article should be addressed to Stephen R. Shirk, Department of Psychology, University of Denver, Denver, Colorado 80208. E-mail: sshirk@nova.psy.du.edu coalesce around a unifying relational construct such as the therapeutic alliance. Instead, a range of relationship variables such as therapy bond, treatment involvement, and perceptions of therapist warmth has been evaluated in relation to outcome. Although several studies have revealed moderate associations among these variables (Estrada \& Russell, 1999; Shirk \& Saiz, 1992), it is likely that they capture varied facets of the therapeutic relationship. Because of the emergent state of the child process literature, a wider set of relationship variables was included in this review than in previous meta-analyses of alliance-outcome relations. To make a direct comparison with the adult alliance literature, instruments were classified as alliance measures or general relationship measures, and associations with outcomes were computed for each.

From a developmental perspective, it has been suggested that the therapeutic relationship may be more critical in child than adult therapy (Shirk \& Saiz, 1992). Because children rarely refer themselves for treatment, often do not recognize or acknowledge the existence of problems, and frequently are at odds with their parents about the goals of therapy, alliance formation can be a formidable challenge with youth (Shirk \& Russell, 1998). Further, the developmental trend toward increasing autonomy from adults can represent an additional obstacle to the formation of a positive therapeutic relationship among adolescents (DiGiuseppe, Linscott, \& Jilton, 1996). Given these considerations, the contribution of the therapeutic relationship to outcome could vary as a function of developmental level.

In addition to developmental level, type of emotional or behavioral problems could moderate the importance of the therapy relationship for treatment outcome (DiGiuseppe et al., 1996; Shirk \& Saiz, 1992). Youngsters with internalizing problems may form a therapeutic relationship more readily than their externalizing counterparts because of greater motivation to reduce internal dis-

\footnotetext{
${ }^{1}$ The study includes samples of children and adolescents; "child" or "children" is used in reference to youths under age 18 unless otherwise noted.
} 
comfort and fewer problems with authority figures (DiGiuseppe et al., 1996). Consequently, the formation of a positive therapeutic relationship is likely to be more challenging, and potentially more critical, for treatment outcome among youngsters with externalizing than internalizing problems. Consistent with this perspective, Eltz, Shirk, and Sarlin (1995) found that adolescents with relationship problems and more negative interpersonal expectations had greater difficulty with alliance formation than comparable teens without such problems and expectations. In turn, alliance formation was strongly predictive of outcome for this group. In contrast, Kendall (1994) found minimal associations between alliance and outcome in a sample of anxiety disordered youths, in part, because of limited variability in alliance scores that were highly positive. Although Martin et al. (2000) did not find alliance-outcome relations to vary as a function of problem type in their adult therapy sample, it is possible that type of problem could moderate the association between therapeutic relationship and outcome in younger samples.

Relatively little is known about the role of relationship processes in different types of child therapy. Nonbehavioral child therapies have emphasized the importance of the therapeutic relationship as a pivotal change mechanism (Shirk \& Russell, 1996), whereas behaviorally oriented treatments have underscored the importance of the therapeutic relationship for treatment collaboration and motivation (Kendall, 1991; Stark, Swearer, Kurowski, Sommer, \& Bowen, 1996). In fact, nonbehavioral child therapies often posit relationship processes as the primary change mechanism, whereas behavioral treatments consider such processes in the context of other active interventions (Shirk \& Russell, 1996). Early studies revealed that relationship variables were moderately associated with outcomes in nonbehavioral child therapy (Taylor, Adelman, \& Kaser-Boyd, 1986; Truax, Altman, Wright, \& Mitchell, 1973) but that relationship variables showed an uneven association with outcomes in CBT (Braswell, Kendall, Braith, Carey, \& Vye, 1985; Kendall, 1994). Thus, it is possible that the strength of therapeutic relationship-treatment outcome associations will vary as a function of type of therapy.

Because children are developing both adaptive and maladaptive behavior in the context of the family (Sameroff, 1994), child treatment often directly involves other family members. Reviews of psychotherapy practice have shown that it is common to include parents and siblings in child treatment, in the form of either family therapy or parent management training (Ollendick \& Russ, 1999). In the latter, direct contact with the child can be relatively limited; consequently, the primary therapeutic relationship in this form of child treatment is between therapist and parent(s). Some years ago Pinsoff (1994) argued that the alliance had been overlooked outside of individual therapy. However, there is growing recognition of the role of the alliance in family therapy (Friedlander, Wildman, Heatherington, \& Skowron, 1994), as well as in parent management training (Kazdin \& Wassell, 1999; Morrissey-Kane \& Prinz, 1999). Although previous meta-analyses of alliance-outcome relations have focused on individual psychotherapy, this relatively narrow scope is not developmentally sensitive to variations in child treatment. Therefore, the relative contribution of the therapeutic relationship to outcome across different modes of therapy, specifically, across individual, parent, and family therapies, is considered.

A related issue concerns the importance of relationship variables across treatments that vary in terms of specific treatment tasks. It is possible that increased structure through manualization decreases the role of nonspecific factors, such as the therapeutic relationship, by increasing the contribution of specific interventions to treatment outcome (Krupnick, Sotsky, Elkin, Watkins, \& Pilkonis, 1996). One factor contributing to variation in structure involves the use of a treatment manual for specifying treatment tasks and organizing the delivery of therapy (Weisz, Donenberg, Han, \& Kauneckis, 1995). Treatments were classified in terms of level of structure on the basis of their use of a treatment manual, and differences in relationship-outcome associations were evaluated across levels of structure.

The context of treatment could moderate the association between relationship variables and outcomes. There is growing evidence that attrition rates from community-based child therapy, estimated between $40 \%$ and $60 \%$ (Armbruster \& Kazdin, 1994; Gould, Shaffer, \& Kaplan, 1985), are substantially higher than rates found in research trials (Brent et al., 1997; Kendall \& Sugarman, 1997). Early dropout and sporadic involvement are likely to dilute treatment strength and compromise effectiveness. Emerging research has indicated that the therapeutic alliance plays a significant role in attrition from child therapy (Kazdin, Holland, \& Crowley, 1997; Shirk, 2001). Consequently, a strong therapeutic relationship appears to be essential in clinic-based therapy to ensure that patients receive an adequate dose of treatment. Treatment context, that is, whether the treatment was conducted in the context of an ongoing clinical service or as part of a research trial, was considered as a potential moderator of relationship-outcome associations.

Like other child process research, studies in this area vary substantially in methodological quality (Russell \& Shirk, 1998). Previous meta-analyses of child therapy outcome have shown that methodological quality is associated with effect size estimates (Shirk \& Russell, 1992; Weiss \& Weisz, 1990). Measures of alliance and similar relationship constructs in child therapy can differ in terms of reliability, source, and overlap with outcome measures. Horvath and Symonds (1991) found that strength of alliance-outcome relations varied as a function of source of measurement. Building on this finding, the impact of timing, source, and shared versus nonshared source of measurement on the strength of relationship-outcome associations was evaluated. Similarly, outcome measures differ in terms of content and source. Previous meta-analyses of child therapy outcomes have revealed that measurement content and source contribute to variations in effect size estimates (Casey \& Berman, 1985; Weisz, Weiss, Alicke, \& Klotz, 1987; Weisz, Weiss, Han, Granger, \& Morton, 1995). These two measurement characteristics were evaluated as potential moderators of the association between therapeutic relationship variables and treatment outcomes. Finally, process studies are conducted in the context of controlled or uncontrolled trials, and these trials can vary substantially in the degree to which they demonstrate change. Both characteristics were considered as potential moderators. Overall, then, a broad set of methodological characteristics were considered in relation to estimates of therapeutic relationship-outcome effects to highlight promising directions as well as significant limitations in existing research.

The current study addressed two major questions. First, what is the overall strength of association between therapeutic relationship variables and outcomes in child psychotherapy? Second, are these associations moderated by substantive or methodological factors? Six variables were considered as possible substantive moderators, 
two patient characteristics and four treatment characteristics. Patient characteristics included the child's age and type of presenting problems; treatment characteristics included type of treatment, mode of treatment, level of structure of treatment, and context of treatment. Seven variables were considered as possible methodological moderators, including timing and source of relationship measurement, content and source of outcome measurement, shared versus cross-source measurement of relationship and outcome variables, and finally, study design and degree to which study treatments produced beneficial effects. To address these issues, we conducted a meta-analysis of extant child process research relating therapeutic relationship variables to treatment outcomes.

\section{Method}

\section{Selection of Studies}

This meta-analysis included 23 studies (18 published articles and 5 unpublished doctoral dissertations). The following procedures were used to identify these studies.

Inclusion criteria. The inclusion criteria for this study were the same as those used by Martin et al. (2000) and Horvath and Symonds (1991) with three exceptions. Consistent with prior meta-analyses in this area, criteria included: (a) the study included a quantifiable measure of association between a therapeutic relationship variable and a measure of outcome; (b) the study needed to be clinical, not analogue; (c) the study needed to include at least five patients; (d) the study needed to be presented in English; (e) and the study was either published or available for review from Dissertation Abstracts (http://wwwlib.umi.com/dissertations).

Our inclusion criteria differed in three ways from those of Martin et al. (2000) and Horvath and Symonds (1991). First, a wider set of relationship variables was included for examination. Although some studies referred to relationship variables as the alliance, most did not. Only those studies that explicitly evaluated some aspect of the therapeutic relationship in association with outcome were included. Independent judges agreed in all cases; otherwise the study was excluded. Second, treatment was not restricted to individual therapy but included family therapy and parent management training as well. Although these treatments differ in terms of participants, they represent common forms of child and adolescent treatment. Separate analyses were conducted to evaluate the strength of relationship-outcome associations for child patients only (excluding other participants) across different modes of therapy. Third, previous meta-analyses required that process and outcome measures be separated in time. Both prospective and concurrent associations were evaluated in this meta-analysis, and strength of association as a function of timing of measurement was evaluated. Subsequent analyses considered the comparability of studies that met all criteria used by Martin et al. (2000) with those that did not. The most common reason for exclusion of a study was the absence of a measure of treatment outcome. Studies that only examined the association of relationship variables with session quality or impact were excluded.

Literature review. To identify relevant articles, several methods of search were completed. First, a literature review was conducted using the following databases: PsycINFO (http://spider.apa.org/psycinfo/index.cfm), ERIC (www.eric.ed.gov), Medline (www.ncbi.nlm.nih.gov), and Dissertation Abstracts. To constrain the search to child and adolescent treatment, child and adolescent were used in conjunction with the words alliance, therapeutic relationship, and outcome. Second, we examined the list of studies included in two previous reviews of child treatment processes (Russell \& Shirk, 1998; Weisz et al., 1998). Third, four journals (Journal of Abnormal Child Psychology, Journal of Clinical Child Psychology, Journal of Consulting and Clinical Psychology, and Journal of the American Academy of Child and Adolescent Psychiatry) were searched by hand to ensure that articles published after the previous reviews were not missed. These steps produced a pool of 38 studies, 23 of which met criteria. All studies were conducted with children under the age of 18 or with the parents and family members of an identified patient under the age of 18

\section{Coding Procedure}

All studies were coded independently for sample, design, and treatment characteristics by Stephen Shirk and Marc Karver. Interrater agreement (kappa) is reported for the independent codes. Disagreements were resolved by discussion between coders.

Patient age. Studies were classified as involving children or adolescents. Studies were categorized as child treatment if the mean age was reported (or could be computed) as under 13 years and as adolescent treatment if mean age was 13 years or older $(\kappa=.92)$.

Type of problems. Each study was coded for the predominant broadband emotional or behavioral problems represented in the sample. Samples were coded as externalizing if the predominant problems involved disruptive behavior disorders or delinquency; as internalizing if the predominant problems involved anxiety, depression, and low self-esteem; and as mixed if no predominant problem was evident in the sample. Only a limited number of studies (2) reported specific diagnoses, instead most relied on scores from self-, parent-, or teacher-report or from therapist description to define the sample. Kappa for type of problem was .64.

Treatment type and mode. Following Weisz, Weiss, et al. (1995), studies were categorized as behavioral or nonbehavioral. Behavioral treatments included CBT, parent training, and skills training interventions; nonbehavioral included psychodynamic, client-centered, and eclectic therapies $(\kappa=.87)$. Each treatment also was coded as individual, family, or parent training. We coded the treatment as individual therapy if the child or adolescent was seen alone with the therapist; as family therapy if the identified patient and other members of the family were seen conjointly in sessions; and as parent training if parents alone were seen by the therapist (although the child might be in collateral treatment). There were no disagreements between coders $(\kappa=1.0)$.

Target relationship. Because some treatments involved multiple participants, target relationship was coded. Target relationship refers to the specific participants in the rated relationship, child-therapist (C-T), parenttherapist (P-T), family-therapist (F-T). Codes are made independent of type of treatment. For example, C-T was coded in family therapy if the rated relationship was between the child and family therapist. Similarly, P-T was coded in family therapy if the rated relationship was between a parent and the therapist, regardless of the presence of other participants. F-T included only those cases in which the average correlation between a group of family members and therapist was reported. There were no disagreements between coders, $\kappa=1.0$.

Level of treatment structure. As an index of level of structure, treatments were coded as either manualized or nonmanualized. To be classified as a manualized treatment, the study needed to report the use of a specific treatment manual and a method for ensuring adherence. It was assumed that manualized treatments involve a higher degree of treatment structure There were no disagreements across raters, $\kappa=1.0$.

Treatment context. Treatments were classified as service therapy or research therapy. To be coded as service therapy, the treatment had to have been part of an existing, clinical service and the assessment of relationship variables and outcomes was added to the ongoing service. Such treatments are often treatment as usual (TAU) and are not modified for research purposes. To be coded as research therapy, the treatment had to have been specifically set up for evaluation and research and not part of an ongoing clinical service. Such treatments are often clinical trials or demonstration projects that include process measures. Interrater agreement as assessed with kappa was .90

Comparability coding. To make direct comparisons with the adult psychotherapy literature, studies were coded in terms of whether they satisfied additional inclusion criteria used by Horvath and Symonds (1991) and Martin et al. (2000). Three criteria were used. First, relationship variables were coded as either alliance or general relationship variables. To be classified as an alliance variable, the measure needed to be referred to 
as therapeutic alliance, working alliance, helping alliance, therapeutic bond, or simply, alliance. Second, the studies needed to involve individual therapy as defined above. Third, the measurement of alliance and outcome needed to be separated in time. Studies that fulfilled all three criteria were classified as alliance studies. All other studies were coded as relationship studies. Interrater agreement as assessed by kappa was .50, $p<.01$. Although kappa was lower for this coding dimension than others, it should be noted that percentage agreement was .86 .

Other methodological characteristics. Studies were coded for a set of methodological and design characteristics. Specifically, source (child, treatment provider, family member) and timing (early, late, or follow-up) of relationship measures, domain (symptoms, global functioning, other) and source of outcome measures were recorded. Of particular concern was whether associations between relationship and outcome variables shared source and/or timing of measurement. In addition, the design (controlled vs. uncontrolled) and degree of change reported (statistically significant vs. nonsignificant) were categorized. Finally, reliabilities for relationship measures, date of publication, and published or unpublished status were recorded.

\section{Calculation of Effect Sizes}

Because most studies in this area of research report results as correlations, the product-moment correlation coefficient $r$ was used as our effect size estimate. This was the statistic used in both meta-analyses of the adult alliance to outcome literature. All results were converted to Fisher's Z equivalents for comparability and to adjust for bias of the $r$ distribution (Hedges \& Olkin, 1985). To find the overall relationship to outcome association, we weighted the effect size in proportion to the number of subjects involved in the study. The formula used was

$$
\mathrm{E}_{\mathrm{sw}}=\text { Sum of }\left[\left(\mathrm{n}_{i}-3\right) * \mathrm{Z}_{r i}\right] / \text { Sum of }\left(\mathrm{n}_{i}-3\right)
$$

where $\mathrm{n}_{i}$ is the sample size for the $i$ th study and $\mathrm{Z}_{r i}$ is the Fisher's transform of the effect size $r$ for the $i$ th study. ${ }^{2}$ After these computations, results were reconverted to $r$ s for ease of interpretation. Thus, effect sizes presented in the Results section refer to $r$ values unless otherwise noted.

When relevant statistics were not reported in the published article or dissertation, we attempted to contact the author(s) to obtain the missing information. In those cases in which we were not successful, reports of nonsignificant effects unaccompanied by any statistic were assigned an effect size of zero.

Combination of effect sizes. In this meta-analysis, we computed weighted effect sizes by sample size and unweighted effect sizes. The reason we calculated both was because of the substantial impact of one study that had a sample that was almost half the size of all other studies combined. In most of the studies in our sample, more than one relationship-outcome association was reported. To correct for a potential weighting bias related to unequal number of associations across studies, we averaged the relationship-outcome associations to form an overall effect size for each study. In addition, the overall relationship-outcome association was disaggregated by source and timing of relationship measurement and by source, content, and specificity of outcome measurement and reanalyzed separately. When data from a study were reported in more than one source (dissertation, chapter, journal), we located and considered all sources of information to prevent duplication of results.

\section{Results}

\section{Sample Characteristics}

The 23 studies that met inclusion criteria are shown in Table 1. These studies were reported over a 27-year period (see Table 1); however only 4 were conducted prior to 1990 . The sample included 18 published and 5 unpublished doctoral dissertations. The mean sample size was 82.22 clients $(S D=146.66)$. However, this mean sample size was inflated because of one study (Noser \& Bickman, 2000) that had a sample size of 731. A more accurate representation of the typical sample size was the median of 47 . The average length of treatment could not be calculated because of variations in reporting across studies. However, a rank ordering of the studies indicated that the median length of treatment was approximately 19 sessions. Unlike adult alliance studies in which the majority of participants are women (Martin et al., 2000), 65\% of the patients in these child and adolescent studies were boys. The mean number of therapists per study (only 13 studies provided this information) was $10.62(S D=6.98)$.

Type of patient. Fourteen studies were conducted in outpatient settings (clinic, school based, or university based). Patients in other studies received in-home family therapy, residential treatment, inpatient treatment, and mentoring. In total, 1,406 patients received some form of outpatient clinic treatment, whereas 485 received other forms of treatment. Parents and/or other family members were included in or were the primary focus of treatment in 8 studies. The diagnostic characteristics of the sample were extremely heterogeneous and ranged from children with anxiety disorders to adolescents adjudicated for delinquent behaviors. Based on our coding for predominant broad band problems, 5 studies involved externalizing samples, 4 involved internalizing samples, and 14 involved mixed samples.

Alliance and relationship scales. The sample of studies used numerous alliance/therapeutic relationship scales with very little overlap in measures across studies. Most of the studies used just one type of therapeutic relationship measure $(n=15)$, however, eight studies attempted to measure the therapeutic relationship with multiple measures. There was no most common measure used, showing the lack of uniformity in the child-adolescent treatment process literature. Some studies measured the family therapeutic alliance (L. N. Johnson, 1998; Van Orman, 1996); others used modified adult measures such as the Working Alliance Inventory (Florsheim, Shotorbani, Guest-Warnick, Barratt, \& Hwang, 2000), others used general relationship measures such as the Barrett-Lennard Relationship Inventory (McNally \& Drummond, 1973) or the Network of Relationships Inventory (Cavell \& Hughes, 2000), and in several studies researchers developed their own measures of therapeutic relationship (Green, 1996; Kendall et al., 1997).

Patients, therapists, parents, family members, and observers made therapeutic relationship ratings. Twelve studies used more than one type of judge. Therapeutic providers $(n=13)$ were the most common, followed by children and/or adolescents $(n=12)$, parents $(n=7)$, observers $(n=2)$, and family members $(n=2)$.

Outcome measures. A wide variety of outcome measures were used in these studies. Fifteen studies measured symptomatology, 4 measured family functioning, 11 used ratings of global functioning, and 11 studies used a variety of measures that could not be easily categorized, such as interpersonal relations, new criminal charges, positive behavior, etc. Patients, therapists, parents, family

\footnotetext{
${ }^{2}$ For example, assume there are three studies with sample sizes of 10 , 20, and 30 participants and effect sizes per study of .2, .4, and .6, respectively. Without weighting by sample size, the mean effect size is .4 . With weighting, the effect size is .2 (7) $+.4(17)+.6(27)$ divided by the sum of all participants across studies $(10-3)+(20-3)+(30-3)$. The weighted effect size, then, would equal .48 .
} 


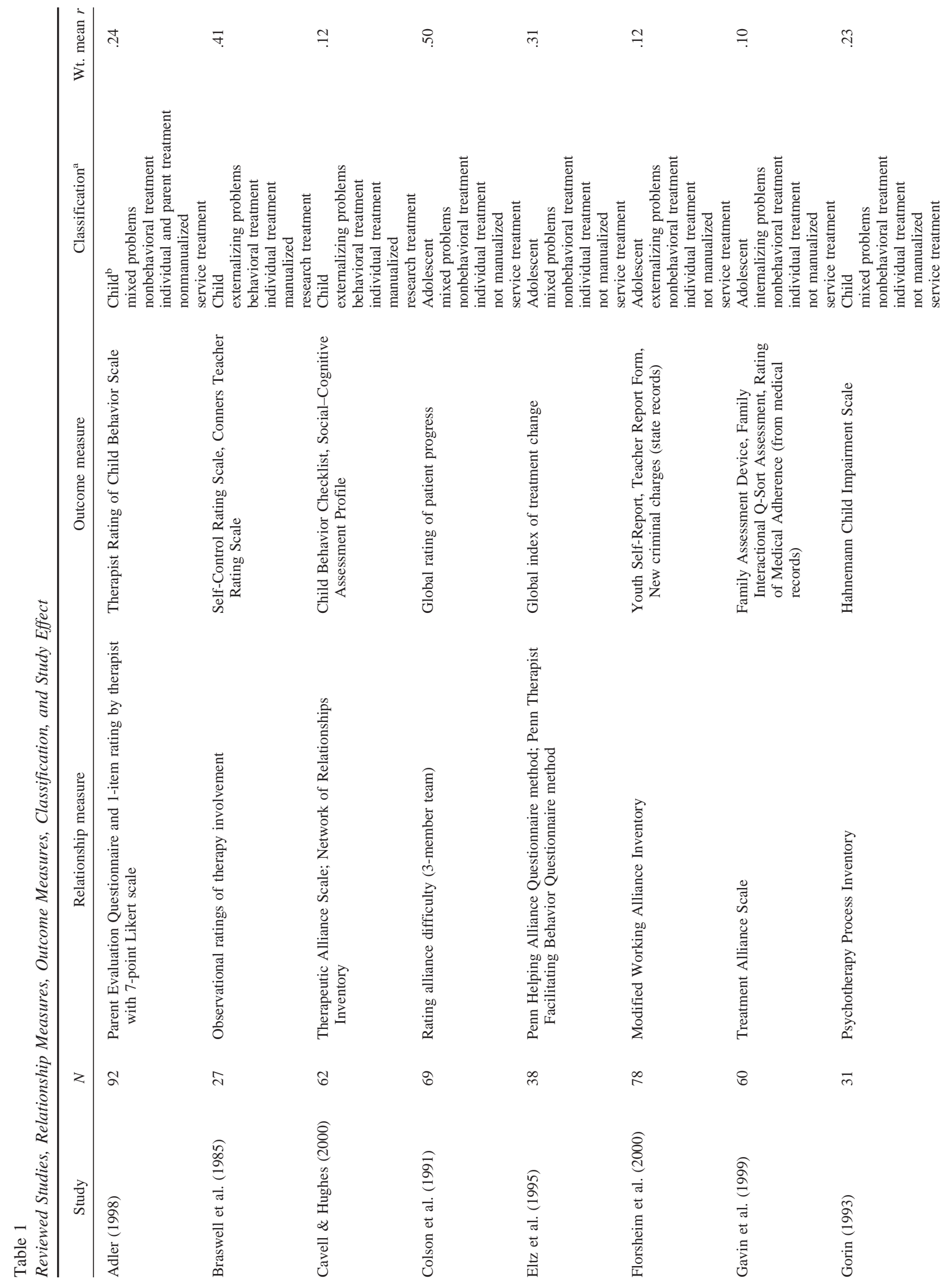




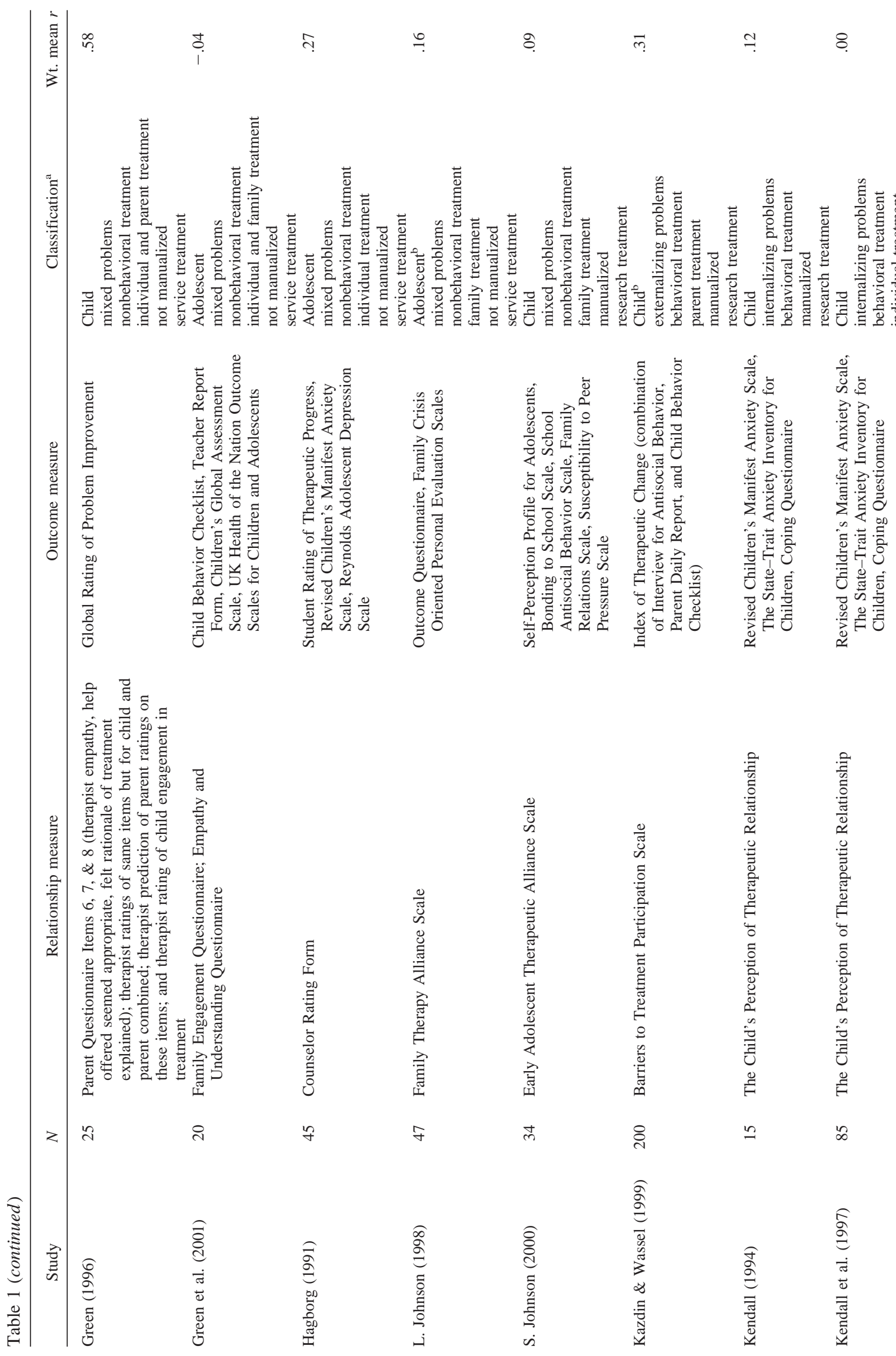




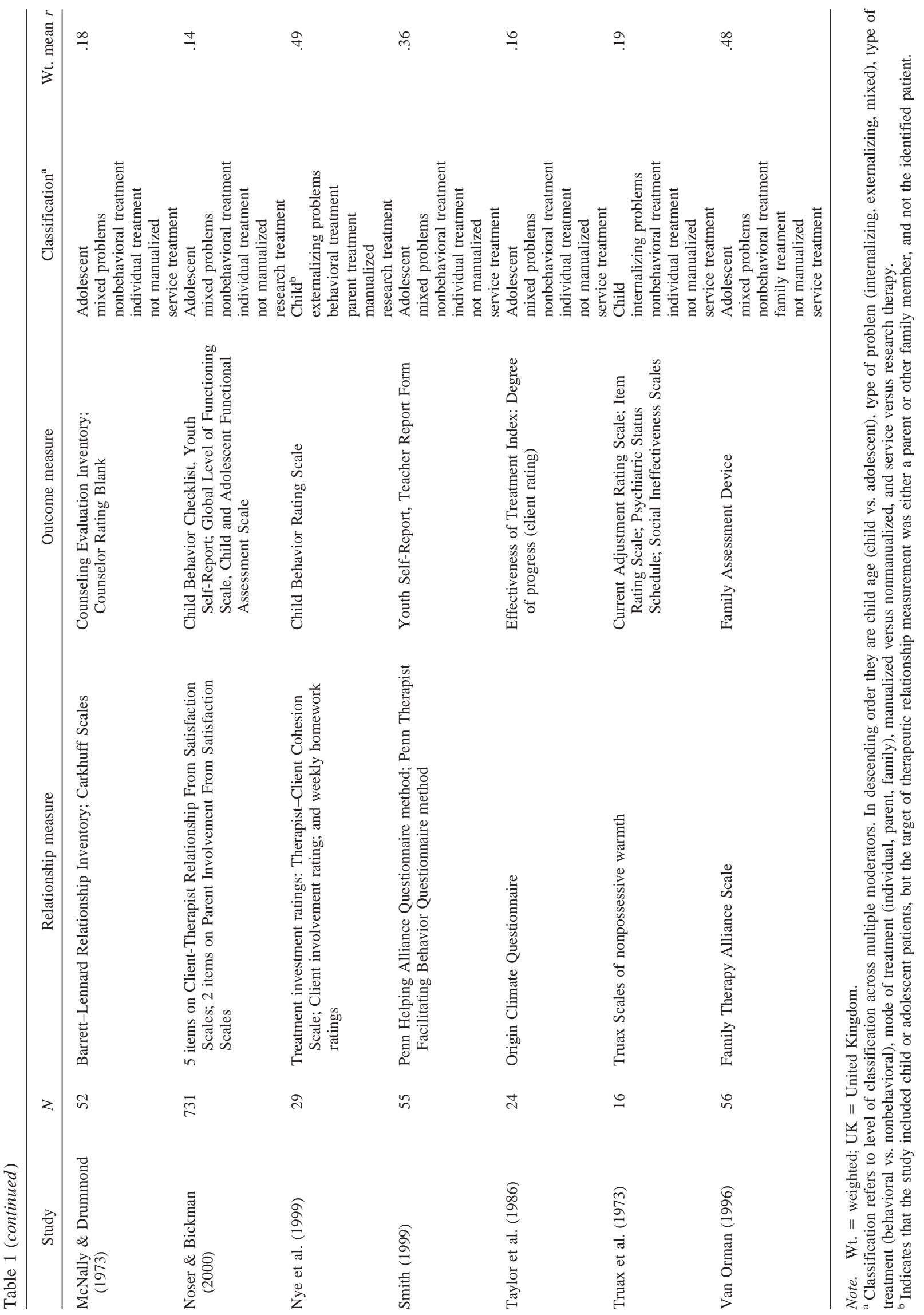


members, or observers completed outcome measures. Fifteen studies included more than one type of respondent. Children and/or adolescents were the most common $(n=13)$, followed by therapeutic providers $(n=12)$ and parents $(n=10)$, observers $(n=5)$, family members $(n=2)$, an objective indicator $(n=1)$, and an average of all informants $(n=1)$. This wide variety of respondents for alliance and outcome measures resulted in 20 different relationship to outcome scoring combinations.

\section{Reliability}

Before proceeding with an examination of relationship to outcome associations, the reliability of alliance/relationship measures was examined. Reliability information was provided by 19 of 23 studies. Most of the studies $(n=15)$ reported Cronbach's alpha as the indicator of reliability. For these studies the average alpha was $.86(S D=.07)$, which is considered acceptable according to Nunnally's (1978) criteria on internal consistency. As point of comparison, in a recent meta-analysis of the adult alliance literature, the average Cronbach's alpha was .87 (Martin et al., 2000).

Interrater reliability was reported in eight studies. An average reliability could not be reported for this category as different statistics were used that do not have equivalent meaning for the same value (percentage agreement, Pearson's $r$, and interclass correlation). The average Pearson's correlation across six studies was .70, which is minimally acceptable (all criteria according to Cooper \& Hedges, 1994; and Nunnally, 1978). One study reported a percentage agreement of .96 that is highly acceptable; another reported an interclass correlation of .34 that is unacceptably low.

Finally, test-retest reliability was reported in two studies that had an average correlation of .83 , which is very good according to criteria established by Bickman et al. (1998). Unfortunately, time interval was reported in only one study.

\section{Relation Between Alliance and Outcome}

The product-moment correlation coefficient $r$ was used as the estimate of effect size (mean $r=.24$; mean $r_{\mathrm{W}}=.20$; estimated population variance $=.01$ ) . The weighted mean correlation is very similar to findings obtained in the most recent meta-analysis of alliance-outcome relations among adults, weighted $r=.22$ (Martin et al., 2000). When converted to $Z$ scores, the unweighted effect size was .25 and the weighted effect size was .21 . The sample in the study by Noser and Bickman (2000) was almost equal to the total sample of the other 22 studies combined, thereby resulting in undue influence. Removing this study from the calculation resulted in an unweighted effect size of .26. In 3 studies, some associations were reported as nonsignificant, but adequate data for effect size estimation were not provided. Removing studies that required a conservative estimate of zero yielded an overall weighted effect size of .22. ${ }^{3}$ According to Cohen's (1992) criteria, these estimates represent small effect sizes similar to those found in the adult literature.

A test of homogeneity was conducted to determine whether effect sizes came from a common pool of effects or whether there were distinct clusters of effect sizes in the sample of studies. The results suggested that there is more variability among these results than might be expected on the basis of chance alone, $Q(22)$ $=39.81, p<.02$. Thus, it is likely that variation was not due to sampling error but possibly because of the existence of more than one population effect. This suggested that there are moderator variables within the sample of relationship-outcome associations, thereby justifying further analyses.

\section{Moderator Analyses}

A number of moderator variables were examined in an attempt to identify potential homogeneous groupings within this heterogeneous collection of studies. The hypothesized moderators fell into two categories: substantive moderators (moderators related to characteristics of patients or treatments) and methodological moderators (moderators related to characteristics of studies). All tests of moderation were conducted by comparing effect sizes across levels of the hypothesized moderator using either independentsample $t$ tests or Wilcoxon's signed rank tests. The Wilcoxon's was used in instances that included both independent and dependent (results from the same study) data. Because of sample size, it was necessary to use this strategy rather than compare only those studies with independent data.

\section{Substantive Moderators}

Patient characteristics. It was hypothesized that patient age might moderate the association between therapeutic relationship and outcome. This result was not found. The association between therapy relationship and outcome for children was $M=.28, S D=$ .33 and for adolescents was $M=.25, S D=.17$, but it was not significant because of substantial variability in effect sizes across studies, $t(17)=.31, p=.761$.

Type of problem was examined as a second patient characteristic. It was hypothesized that studies with externalizing children would show a stronger association between relationship and outcome than would studies with internalizing children. Results, in fact, supported this hypothesis $\left(M_{\text {internalizing }}=.10, S D=.08\right.$, $M_{\text {externalizing }}=.30, S D=.18$ ), and were reliably different, $t(7)=2.00, p<.05$.

Treatment characteristics. It was hypothesized that the therapeutic relationship-outcome association might be moderated by type of treatment because of different functions of the relationship in these two forms of therapy. However, this was not found, $M_{\text {nonbehavioral }}=.25, S D=.18 ; M_{\text {behavioral }}=.26, S D=.20$, $t(21)=0.08, p=.47$. These results suggest that the association of therapy relationship with treatment outcome is comparable across behavioral and nonbehavioral child treatments.

The association of the therapy relationship with outcome across individual, family, and parent treatments was also examined. Mode of treatment did not moderate relationship-outcome associations. Although the association appeared to be strongest for parent treatments $\left(M_{\text {parent }}=.33, S D=.13\right)$, this relation did not reliably differ from means for family $\left(M_{\text {family }}=.24, S D=.20\right)$ or individual therapy $\left(M_{\text {individual }}=.22, S D=.17\right) ; F(2,20)=0.17$, $p=.50$.

Because some treatments involved multiple participants, mean associations were computed by relationship target to distinguish the potential effect of rated relationship (child, parent, family)

\footnotetext{
${ }^{3}$ Because the study by Truax et al. (1973) reported unacceptably low reliability for their therapeutic relationship measure, overall effects were computed without this study. The mean weighted correlation for the sample of studies was 24 .
} 
from mode of treatment (individual, parent training, family therapy). Associations did not differ significantly, $M_{\text {child }}=.27, S D=$ $.24 ; M_{\text {parent }}=.23, S D=.26 ; \mathrm{M}_{\text {family }}=.34, S D=.26$. Only two correlations were reported for the family therapeutic relationship.

It was hypothesized that the therapy relationship would account for greater variance in outcome in nonmanualized than in manualized treatments insofar as nonmanualized treatments rely on nonspecific factors for change and manualized treatments focus on specific treatment tasks. Results did not support this hypothesis. Instead, the association between relationship variables and outcomes was essentially the same across level of treatment structure $\left(M_{\text {manualized }}=.23, S D=.20 ; M_{\text {nonmanualized }}=.24, S D=.15\right)$, $t(21)=0.16, p=.44$. It is noteworthy that this analysis was confounded with type of treatment. In fact, only one manualized treatment was nonbehavioral.

Finally, context of therapy was considered as a moderator. It was hypothesized that relationship variables might be more critical in service therapy than research therapy because of its potential role in ensuring an adequate dose of treatment in service settings. Though in the predicted direction, the results were not reliable, $M_{\text {service }}=.27, S D=.19 ; M_{\text {research }}=.22, S D=.18, t(57)=0.57$, $p=.29$.

\section{Methodological Moderators}

The set of studies included in this meta-analysis varied in terms of when they measured the therapy relationship, who reported the relationship and outcomes, and what type of outcomes were assessed. In addition, studies varied in terms of design, and study treatments differed in the degree to which they produced significant effects. These methodological factors were considered as potential moderators of relationship-outcome associations.

Therapeutic relationship measurement. Relationship variables varied in terms of timing and source of measurement. For timing, relationship variables were assessed early in treatment (first third of treatment) or late in treatment (last third, at termination, or following termination). Results revealed a significant effect for time of measurement, with late measures showing larger associations $\left(M_{\text {late }}=.27, S D=.22\right)$ than early measures $\left(M_{\text {early }}=.12\right.$, $S D=.08), z(18)=3.51, p<.01$.

Relationship variables were assessed from multiple perspectives including child patient, therapist or treatment provider, parent, and other family members. There was some variation by source of measurement, child and/or adolescent report $(M=.18, S D=.14)$, treatment provider $(M=.29, S D=.18)$, parent and/or family member $(M=.26 ; S D=.22)$. In contrast to results from the adult literature, associations between relationship and outcome were stronger for therapist and/or provider reports than for child patient reports, $Z(13)=3.06, p<.01$. Child reports showed very limited variability, reflecting the tendency of children to report positive therapeutic relationships.

Outcome measurement. Outcome variables varied in terms of measurement domain and source. For domain, outcomes were classified as symptom measures, global functioning measures, and other measures. Relative to measures of symptoms $\left(M_{\text {symptom }}=\right.$ $.21, S D=.19)$ or other measures $\left(M_{\text {other }}=.20, S D=.24\right)$, measures of global functioning $\left(M_{\text {global }}=.32, S D=.20\right)$ were more highly associated with relationship variables, $Z(15)=2.16$, $p<.04, Z(15)=2.94, p<.01$, respectively for each comparison. Means did not differ for symptom measures and other measures.
Outcome measures also varied by informant. Sources were classified as self $(n=10)$, parent $(n=13)$, treatment provider $(n=$ $12)$, and observer $(n=5)$. The only significant difference was between self-reports $\left(M_{\text {self }}=.17, S D=.13\right)$ and provider reports $\left(M_{\text {provider }}=.26, S D=.19\right)$ of outcomes, $Z(13)=2.167, p<.03$; all other comparisons were not reliably different $\left(M_{\text {parent }}=.23\right.$, $S D=.17 ; M_{\text {observer }}=.21, S D=.17$ ).

Shared versus cross-source associations. The possibility that associations could be moderated by shared versus cross-source reporting of relationship and outcome variables was examined. In fact, this methodological factor did make a difference with shared source associations $\left(M_{\text {shared }}=.29, S D=.21\right)$ greater than crosssource associations $\left(M_{\text {cross-source }}=.19, S D=.19\right), z(19)=3.82$, $p<.01$.

Study design and effects. The studies included in this metaanalysis varied in terms of design and overall treatment effects. In fact, many of the treatments that were delivered have no or very little evidence for their efficacy. Thus, beneficial treatment effects could not be assumed for all studies. One concern was that ineffective treatments might yield limited variability in outcomes, thereby attenuating associations with relationship variables. Studies were classified into three categories: controlled trials that failed to produce treatment effects (CTF, $n=3$ ), controlled trials that produced positive effects (CTP, $n=4)$, and uncontrolled trials that produced significant pre-posttreatment effects (NCP, $n=16$ ). There were no uncontrolled process studies that failed to produce significant pre-posttreatment effects. Given the small number of studies, it is not surprising that differences across these groups failed to reach significance. However, there was a pattern suggesting that treatments with positive outcomes yielded the largest associations between relationship variables and outcomes, $M_{\mathrm{CTF}}=$ $.12, S D=.02 ; M_{\mathrm{NCP}}=.27, S D=.25 ; M_{\mathrm{CTP}}=.28, S D=.25$.

Comparability analyses. To draw a direct comparison with results from meta-analyses of alliance-outcome relations in the adult literature, we classified the 23 studies into two groups, those that met full inclusion criteria used by Horvath and Symonds (1991) and Martin et al. (2000) and those that did not. Remarkably, only 1 study (Eltz et al., 1995) met criteria, and the prospective association between alliance and outcome was very small, mean $r=.08$. All other studies failed to meet criteria because of three factors; treatments were not individual, the word alliance or bond was not used as the name for the relationship variable, and/or measurements were not prospective.

Additional analyses. Because treatments were delivered in both outpatient clinic and other settings (e.g., residential or inpatient settings), we checked the magnitude of association across settings. Results did not differ; outpatient $r=.25$, other $r=.26$. Finally, publication status and date of publication were examined in relation to magnitude of association between relationship and outcome. Associations from unpublished dissertations did not differ from associations from published articles, $M_{\text {publication }}=.25$, $S D=.19, M_{\text {dissertation }}=.28, S D=.17$. There also was no relation between date of publication and magnitude of association, $r=$ -.08 .

\section{Discussion}

The results of this meta-analysis showed that the therapeutic relationship is related to outcome across diverse types and modes of child treatment. The average relationship-outcome correlation 
was comparable to results attained with adults for allianceoutcome relations (Horvath \& Symonds, 1991; Martin et al., 2000). In fact, the estimated association between therapeutic relationship and outcome in individual child and adolescent therapy was identical to the alliance-outcome estimate with adults. Taken together, results suggest that the therapeutic relationship was modestly associated with outcome, not only across divergent types of treatment but also across levels of development as well. In essence, the therapeutic relationship has a modest, but consistent, association with outcome with children, adolescents, and adults. To be sure, inclusion criteria differed across meta-analyses, but the fact that estimated effects are quite similar despite the inclusion of a wider range of treatments and relationship variables in this review suggests that the role of the therapeutic relationship is reasonably robust and consistent.

The strength of these associations should be considered in relation to other predictors of child and adolescent treatment outcome. The absence of research on child therapy process makes it virtually impossible to evaluate the significance of relationship variables relative to other process predictors (Russell \& Shirk, 1998). However, a number of studies have evaluated patient characteristics that account for variations in treatment response (Patterson \& Chamberlain, 1994; Rohde, Clarke, Lewinsohn, Seeley, \& Kaufman, 2001; Southam-Gerow, Kendall, \& Weersing, 2001). For example, child age and high levels of internalizing psychopathology predicted poorer response to CBT of anxiety disorders (Southam-Gerow et al., 2001), whereas adolescent comorbidity predicted symptom reduction but not recovery in group CBT of adolescent depression (Rohde et al., 2001). Effect sizes for such patient characteristics appear to be somewhat larger than therapeutic relationship effects. However, when the full set of pretreatment patient characteristics are considered, as we computed in the study by Southam-Gerow et al. (2001), the mean effect size estimate of .20 is quite similar to the estimate for the therapeutic relationship. ${ }^{4}$

Results revealed that associations between the therapeutic relationship and outcome were moderated by both substantive and methodological factors. Among substantive moderators, type of patient was the only variable that produced reliable differences. Analyses revealed stronger associations for externalizing children than internalizing children. Clinical accounts have noted difficulties with treatment engagement and alliance formation with externalizing children (Henggeler, Schoenwald, Borduin, Rowland, \& Cunningham, 1998). Similarly, research with adults has shown that patient hostility is a predictor of alliance difficulties (Horvath \& Luborsky, 1993). Hostility, along with other interpersonal problems (Eltz et al., 1995), could impede relationship formation among children with externalizing problems. Such problems may be less pronounced in children with internalizing problems. Consequently, the formation of a therapeutic relationship may be both more challenging and more critical for outcome among youth with externalizing rather than internalizing problems. Alternatively, in this sample, externalizing problems were treated with both individual methods and parent management training. It is possible that the stronger association for externalizing rather than internalizing children is confounded with mode of treatment. In fact, the association between therapy relationship and outcome was relatively, but not reliably, higher for parent treatment than individual child treatment. Small sample size limited our ability to disentangle such potential confounds.
Treatment characteristics did not moderate associations between the therapy relationship and treatment outcome. Associations were comparable for behavioral and nonbehavioral treatments; individual, parent, and family treatments; manualized and nonmanualized treatments; and service versus research treatments. The consistency of relationship-outcome associations across these factors parallels results from previous meta-analyses with adults. Neither Horvath and Symonds (1991) nor Martin et al. (2000) found differences in alliance-outcome relations by treatment characteristics. Even the inclusion of modes of therapy as disparate as individual child and family therapy did not yield different results. In this respect, it appears that the therapeutic relationship represents a hardy nonspecific factor in therapy. Although additional research is needed to determine the function of the therapeutic relationship in different types and modes of therapy, these results indicated that relationship constructs are relevant for understanding change in diverse child treatments and varied treatment contexts.

Five methodological factors moderated the association between relationship and outcome variables. Results showed that measures of the relationship obtained late in therapy were more strongly associated with outcomes than measures taken early in therapy. Although findings have not been consistent with adults, this pattern runs in the opposite direction of results obtained by Horvath and Symonds (1991), who found a trend for early alliance to be more predictive of outcome than late alliance. It is possible that relationship formation evolves more slowly with children than adults and that late measures are more reliable indicators of the therapeutic relationship than early measures. More concerning, however, is the possibility that later relationship measures are confounded with outcomes. On the basis of the current findings, there is very little support for a predictive association between relationship variables and outcomes. This represents a significant deficit in the existing child literature on relationship-outcome associations and parallels a major controversy in the adult literature on direction of effects between treatment alliance and treatment progress (Feeley, DeRubeis, \& Gelfand, 1998).

Source of the therapeutic relationship also moderated strength of associations. However, unlike findings in the adult literature that show patient reports to be the best predictor of outcomes (Horvath \& Symonds, 1991), our results suggested that reports from treatment providers were more strongly associated with outcomes than reports from child and adolescent patients. It is possible that child and adolescent patients lack the social cognitive skills to accurately evaluate the therapeutic relationship or tend to be more positively biased in their appraisals than adults. In support of the latter possibility is evidence for restricted range in child reports that tend to cluster near the positive end of ratings (Kendall, 1994; Kendall et al., 1997; Smith-Acuna, Durlak, \& Kaspar, 1991). These results should be interpreted with caution, however. Differences with the adult literature could be a function of differences in types of relationship measures and modes of treatments across respective meta-analytic reviews.

A third methodological moderator involved shared versus crosssource informants. Results indicated that associations between relationship and outcome variables were stronger for shared versus

\footnotetext{
${ }^{4}$ This estimate is based on effect sizes computed for participant measures and posttreatment responses.
} 
cross-source informants. As Horvath and Symonds (1991) have noted, the common assumption among psychotherapy researchers is that a patient or therapist who is satisfied with the process of therapy is likely to rate its results as beneficial. In the context of stronger associations for late versus early measures of the therapy relationship, it is likely that participants who were pleased with progress were inclined to rate process positively. In brief, the association between relationship and outcome in child therapy may be "inflated" by shared measurement source and biased by concurrent perceptions of progress.

Finally, the association between therapeutic relationship variables and treatment outcomes was moderated by outcome domain and outcome source. Relationship variables were most highly correlated with measures of change in global functioning. To the degree that this type of outcome measure includes indicators of interpersonal functioning or social competence, the stronger association could be due to similarity in content domains. Alternatively, given the nonspecific character of many global functioning scales, it is possible that they may be more readily influenced by perceptions of treatment process than are measures of specific symptoms. Overall, few studies in this sample used structured diagnostic interviews or other independent assessments of treatment progress. In fact, the vast majority of outcomes were from participant sources, including parents of treated children, who were not naive to treatment condition or treatment process. This problem may be exacerbated in the case of treatment providers' reports of outcomes. When outcome was measured from the provider perspective, the association between therapeutic relationship and outcome was stronger than when measured from the child's perspective. Providers, as agents of change, are not in a neutral position to evaluate change. Again, it is possible that evaluations of progress could be confounded with perceptions of process.

It was noteworthy that only two measures of the therapy relationship were used in more than one study, the Child's Perception of Therapy Relationship developed by Kendall (1994) and the Penn Helping Alliance Questionnaire developed by Luborsky, McLellan, Woody, O'Brien, and Auerbach (1985) and modified for use with adolescents. Both measures showed adequate internal reliability, and the Penn measure showed moderate temporal stability. These measures may represent a good starting point for investigators who are interested in evaluating the treatment alliance in child and adolescent therapy, respectively. Despite evidence from this review indicating that measures of the therapy relationship were generally reliable, research on child therapy process would benefit from the use of a consistent set of measures across studies. Research with adults has shown that measures of the alliance are not equally related to outcome (Martin et al., 2000); consequently, it is not clear if some of the variation found in this review reflects differences in relationship measures.

A major limitation of this meta-analysis was the limited sample of studies that have examined relationship-outcome associations in child therapy. To establish an estimate of the association between relationship variables and outcomes, it was not possible to restrict the sample to studies that included alliance measures per se; instead, it was necessary to include a range of relationship process variables. In fact, only one study met the full inclusion criteria used in meta-analyses of alliance-outcome relations in adult therapy. Further, it was not possible to evaluate interactions between classification variables; therefore, some potential confounds could not be ruled out. However, it should be noted that the first meta-analysis of alliance-outcome relations in the adult literature (Horvath \& Symonds, 1991) included a similar number of studies $(n=24)$. Subsequently, research on the alliance in adult therapy burgeoned; the most recent meta-analysis (Martin et al., $2000)$ included substantially more studies $(n=79)$, most of which were published after the original review. We hope that this review triggers similar interest in relationship processes in child treatment.

The results of this meta-analysis provide direction for future research on relationship processes in child psychotherapy. First, many of the treatments included in this review had limited evidence of efficacy. Consequently, for some types of treatment, process research has preceded outcome research. Results showed relatively stronger associations between relationship variables and outcomes for treatments that produced beneficial effects, though this trend did not attain statistical significance. Nevertheless, research on therapy processes in treatments with no demonstrated efficacy does not represent a productive direction for child process research. Instead, it makes more sense to conduct analyses of process after a treatment has been shown to be efficacious (Kendall, 2000). An increasing number of child treatments have been shown to be efficacious (Kazdin \& Weisz, 1998), and these treatments should receive priority among process researchers. Conversely, researchers investigating efficacious treatments should make it a priority to consider relationship variables, as well as technical variables, in their search for predictors of outcome.

Second, the potential contribution of relationship variables to outcome in child treatment is ambiguous at this point because measures have not been separated temporally. Too many of the current studies measured the therapy relationship near or at the end of treatment, and results from these studies appear to have inflated relationship-outcome associations. The alliance or other relationship constructs must be measured prior to outcome and preferably at multiple points in time. This direction is necessary if we are to establish a predictive relationship, and understand the course of relationship formation in child treatment.

Third, results of this review indicated that relationship-outcome associations may be inflated by shared source variance. Both therapeutic relationship and outcome should be assessed from multiple perspectives and with multiple methods. Although patient report shows the strongest relationship with outcome in the adult literature (Horvath \& Luborsky, 1993; Horvath \& Symonds, 1991), developmental differences in self-monitoring, perspective taking, and meta-cognition could impact children's ability to report accurately on therapy processes (Shirk \& Russell, 1998). Consequently, it may be especially important to develop observational methods for evaluating relationship constructs in child treatment. It was noteworthy that only two studies used observational measures of the relationship in this review.

Fourth, there was evidence that type of problem moderated the association between relationship and outcome, despite the fact that many of the studies in this review included heterogeneous samples. It is possible that the strength and function of the therapeutic relationship will differ for children with varied disorders. Future process research must take greater care in assessing and identifying homogeneous clinical samples and subsamples.

Finally, in none of the reviewed studies was the contribution of relationship variables to outcome compared with the contribution of other process variables, including collaboration with specific therapy tasks and procedures. As a result, the relative contribution of nonspecific relationship processes and specific therapy tech- 
niques cannot be gauged at this time, nor is it possible to know whether relationship processes such as the alliance bear a direct or indirect association with outcome. It is possible that relationship and technical processes work in concert with a positive relationship priming increased collaboration with treatment tasks (Shirk \& Russell, 1996). Future studies of relationship processes in child treatment should consider other dimensions of process to estimate the unique contribution of the therapeutic relationship to treatment outcome.

\section{References}

References marked with an asterisk indicate studies included in the meta-analysis.

*Adler, A. G. (1998). The alliance between child therapist and parent: How it predicts treatment outcome and reflects parent characteristics. Unpublished dissertation, Yale University, New Haven, CT.

Armbruster, P., \& Kazdin, A. E. (1994). Attrition in child psychotherapy. Advances in Clinical Child Psychology, 16, 81-108.

Axline, V. (1947). Play therapy. New York: Ballantine Books.

Bickman, L., Nurcombe, B., Townsend, C., Belle, M., Schut, L., \& Karver, M. (August, 1998). Consumer measurement systems in child and adolescent mental health. Report to Commonwealth Mental Health Branch, Commonwealth Department of Health and Family Services. University of Queensland, St. Lucia, Queensland, Australia and Vanderbilt University, Nashville, TN.

*Braswell, L., Kendall, P. C., Braith, J., Carey, M. P., \& Vye, C. S. (1985). "Involvement" in cognitive-behavioral therapy with children: Process and its relationship to outcome. Cognitive Therapy and Research, 9, 611-630.

Brent, D., Holder, D., Kolko, D., Birmaher, D., Baugher, M., Roth, C., et al. (1997). A clinical psychotherapy trial for adolescent depression comparing cognitive, family, and supportive therapy. Archives of General Psychiatry, 34, 877-895.

Casey, R., \& Berman, J. (1985). The outcome of psychotherapy with children. Psychological Bulletin, 98, 388-400.

*Cavell, T. A., \& Hughes, J. N. (2000). Secondary prevention as context for assessing change processes in aggressive children. Journal of School Psychology, 38, 199-235.

Cohen, J. (1992). A power primer. Psychological Bulletin, 112, 155-159.

*Colson, D. B., Cornsweet, C., Murphy, T., O’Malley, F., Hyland, P. S., McParland, M., \& Coyne, L. (1991). Perceived treatment difficulty and therapeutic alliance on an adolescent psychiatric hospital unit. American Journal of Orthopsychiatry, 61, 221-229.

Cooper, H., \& Hedges, L. (1994). The handbook of research synthesis. New York: Russell Sage Foundation.

DiGiuseppe, R., Linscott, J., \& Jilton, R. (1996). The therapeutic alliance in adolescent psychotherapy. Applied and Preventive Psychology, 5, $85-100$

*Eltz, M. J., Shirk, S. R., \& Sarlin, N. (1995). Alliance formation and treatment outcome among maltreated adolescents. Child Abuse and Neglect, 19, 419-431.

Estrada, A., \& Russell, R. (1999). The development of Child Psychotherapy Process Scales (CPPS). Psychotherapy Research, 9, 154-166.

Feeley, M., DeRubeis, R., \& Gelfand, L. (1999). The temporal relation of adherence and alliance to symptom change in cognitive therapy for depression. Journal of Consulting and Clinical Psychology, 67, 578582 .

*Florsheim, P., Shotorbani, S., Guest-Warnick, G., Barratt, T., \& Hwang, W. (2000). Role of the working alliance in the treatment of delinquent boys in community-based programs. Journal of Clinical Child Psychology, 29, 94-107.

Freud, A. (1946). The psychoanalytic treatment of children. New York: International Universities Press.

Friedlander, M. L., Wildman, J., Heatherington, L., \& Skowron, E. (1994).
What we do and don't know about the process of family therapy. Journal of Family Psychology, 8, 390-416.

*Gavin, L. A., Wamboldt, M. Z., Sorokin, N., Levy, S. Y., \& Wamboldt, F. S. (1999). Treatment alliance and its association with family functioning, adherence, and medical outcome in adolescents with severe, chronic asthma. Journal of Pediatric Psychology, 24, 355-365.

Goldfried, M. (1998). A comment on psychotherapy integration in the treatment of children. Journal of Clinical Child Psychology, 28, 49-53.

*Gorin, S. S. (1993). The prediction of child psychotherapy outcome: Factors specific to treatment. Psychotherapy, 30, 152-158.

Gould, M., Shaffer, D., \& Kaplan, D. (1985). The characteristics of dropouts from a child psychiatry clinic. Journal of the American Academy of Child and Adolescent Psychiatry, 24, 316-328.

*Green, J. M. (1996). Engagement and empathy: A pilot study of the therapeutic alliance in outpatient child psychiatry. Child Psychology \& Psychiatry Review, 1, 130-138.

*Green, J., Kroll, L., Imrie, D., Frances, F., Begum, K., Harrison, L., \& Anson, R. (2001). Health gain and outcome predictors during inpatient and related day treatment in child and adolescent psychiatry. Journal of the American Academy of Child and Adolescent Psychiatry, 40, 325332.

*Hagborg, W. J. (1991). Adolescent clients and perceived counselor characteristics: A study of background characteristics, therapeutic progress, psychological distress, and social desirability. Journal of Clinical Psychology, 47, 107-113.

Hedges, L. V., \& Olkin, I. (1985). Statistical methods for meta-analysis. New York: Academic Press.

Henggeler, S., Schoenwald, S., Borduin, C., Rowland, M., \& Cunningham, P. (1998). Multisystemic treatment of antisocial behavior in children and adolescents. New York: Guilford Press.

Horvath, A., \& Luborsky, L. (1993). The role of the therapeutic alliance in psychotherapy. Journal of Consulting and Clinical Psychology, 61, 561-573.

Horvath, A., \& Symonds, R. D. (1991). Relation between working alliance and outcome in psychotherapy: A meta-analysis. Journal of Counseling Psychology, 38, 139-149.

*Johnson, L. N. (1998). The therapeutic alliance in home-based family therapy: Is it predictive of outcome? Unpublished doctoral dissertation, Kansas State University, Manhattan.

*Johnson, S. (2000). The therapeutic alliance with early adolescents: Introduction of an instrument. Unpublished doctoral dissertation, Temple University, Philadelphia.

Kazdin, A. E., Bass, D., Ayers, W., \& Rodgers, A. (1990). Empirical and clinical focus of child and adolescent psychotherapy research. Journal of Consulting and Clinical Psychology, 58, 729-740.

Kazdin, A. E., Holland, L., \& Crowley, M. (1997). Family experience of barriers to treatment and premature termination from child therapy. Journal of Consulting and Clinical Psychology, 65, 453-463.

Kazdin, A. E., \& Kendall, P. C. (1998). Current progress and future plans for developing treatments: Comments and perspectives. Journal of Clinical Child Psychology, 27, 217-226.

*Kazdin, A. E., \& Wassell, G. (1999). Barriers to treatment participation and therapeutic change among children referred for conduct disorder. Journal of Clinical Child Psychology, 28, 160-172.

Kazdin, A. E., \& Weisz, J. (1998). Identifying and developing empirically supported child and adolescent treatments. Journal of Consulting and Clinical Psychology, 66, 19-38.

Kendall, P. C. (Ed.). (1991). Child and adolescent therapy: Cognitivebehavioral procedures. New York: Guilford Press.

*Kendall, P. C. (1994). Treating anxiety disorders in children: Results of a randomized clinical trial. Journal of Consulting and Clinical Psychology, 62, 100-110.

Kendall, P. C. (2000). Round of applause for an agenda and report cards for child and adolescent psychotherapy research. Archives of General Psychiatry, 57, 839-840. 
*Kendall, P. C., Flannery-Schroeder, E., Panichelli-Mindell, S. M., Southam-Gerow, M., Henin, A., \& Warman, M. (1997). Therapy for youths with anxiety disorders: A second randomized clinical trial. Journal of Consulting and Clinical Psychology, 65, 366-380.

Kendall, P. C., \& Sugarman, A. (1997). Attrition in the treatment of childhood anxiety disorders. Journal of Consulting and Clinical Psychology, 65, 883-888.

Krupnick, J, Sotsky, S., Elkin, I., Watkins, J., \& Pilkonis, P. (1996). The role of the therapeutic alliance in psychotherapy and pharmacotherapy outcome: Findings in the National Institute of Mental Health Treatment of Depression Collaborative Research Program. Journal of Consulting and Clinical Psychology, 64, 532-539.

Luborsky, L., McLellan, A. T., Woody, G. E., O’Brien, C., \& Auerbach, A. (1985). Therapist success and its determinants. Archives of General Psychiatry, 42, 602-611.

Martin, D. J., Graske, J. P., \& Davis, M. K. (2000). Relation of the therapeutic alliance with outcome and other variables: A meta-analytic review. Journal of Consulting and Clinical Psychology, 68, 438-450.

*McNally, H. A., \& Drummond, R. (1973). Clients' need for social approval and perceptions of counseling relationship and outcomes. Psychological Reports, 32, 363-366.

Morrisey-Kane, E., \& Prinz, R. J. (1999). Engagement in child and adolescent treatment: The role of parental cognitions and attributions. Clinical Child and Family Psychology Review, 2, 183-198.

*Noser, K., \& Bickman, L. (2000). Quality indicators of children's mental health services: Do they predict improved client outcomes? Journal of Emotional and Behavioral Disorders, 8, 9-18.

Nunnally, J. C. (1978). Psychometric theory (2nd ed.). New York: McGraw-Hill.

*Nye, C. L., Zucker, R. A., \& Fitzgerald, H. E. (1999). Early family-based intervention in the path to alcohol problems: Rationale and relationship between treatment process characteristics and child and parenting outcomes. Journal of Studies on Alcohol, Supplement 13, 10-21.

Ollendick, T., \& Russ, S. (1999). Psychotherapy with children and families: Historical traditions and current trends. In S. Russ \& T. Ollendick (Eds.), Handbook of psychotherapy with children and families (pp. 3-13). New York: Kluwer Academic/Plenum Publishers.

Patterson, G., \& Chamberlain, P. (1994). A functional analysis of resistance during parent training therapy. Clinical Psychology: Science and Practice, 1, 53-70.

Pinsoff, W. M. (1994). An integrative systems perspective on the therapeutic alliance: Theoretical, clinical, and research implications. In A. Horvath \& L. Greenberg (Eds.), The working alliance: Theory, research, and practice (pp. 173-195). New York: Wiley.

Rohde, P., Clarke, G., Lewinsohn, P., Seeley, J., \& Kaufman, N. (2001). Impact of comorbidity on a cognitive-behavioral group treatment for adolescent depression. Journal of the American Academy of Child and Adolescent Psychiatry, 46, 795-802.

Russell, R. L., \& Shirk, S. R. (1998). Child psychotherapy process research. Advances in Clinical Child Psychology, 20, 93-124.

Sameroff, A. (1994). Developmental systems and family functioning. In R. Parke \& S. Kellam (Eds.), Exploring family relationships with other social contexts: Advances in family research (pp. 199-214). Hillsdale, NJ: Erlbaum.

Shirk, S. R. (2001). The road to effective child psychological services: Treatment process and outcome research. In J. Hughes, A. LaGreca, \& J. Conoley (Eds.), Handbook of psychological services for children and adolescents (pp. 43-59). New York: Oxford University Press.

Shirk, S. R., \& Russell, R. L. (1992). A reevaluation of estimates of child therapy effectiveness. Journal of the American Academy of Child and Adolescent Psychiatry, 31, 703-709.

Shirk, S. R., \& Russell, R. L. (1996). Change processes in child psychotherapy: Revitalizing treatment and research. New York: Guilford Press.

Shirk, S. R., \& Russell, R. L. (1998). Process issues in child psychotherapy. In A. Bellack \& M. Hersen (Eds.), Comprehensive clinical psychology (Vol. 5, pp. 57-82). T. Ollendick (Vol. Ed.) Children and adolescents: Clinical formulations and treatment. Oxford, England: Pergamon.

Shirk, S. R., \& Saiz, C. (1992). The therapeutic alliance in child therapy: Clinical, empirical, and developmental perspectives. Development and Psychopathology, 4, 713-728.

*Smith, R. D. (1999). Using object relations to predict outcome for adolescents in residential treatment. Unpublished doctoral dissertation, University of Denver, CO.

Smith-Acuna, S., Durlak, J., \& Kaspar, C. (1991). Development of child psychotherapy process scales. Journal of Clinical Child Psychology, 20 , $132-139$.

Southam-Gerow, M., \& Kendall, P. C. (1996). Long-term follow-up of a cognitive-behavioral therapy for anxiety disordered youth. Journal of Consulting and Clinical Psychology, 64, 724-730.

Southam-Gerow, M., Kendall, P. C., \& Weersing, V. R. (2001). Examining outcome variability: Correlates of treatment response in a child and adolescent anxiety clinic. Journal of Clinical Child Psychology, 30, $422-436$.

Stark, K., Swearer, S., Kurowski, C., Sommer, D., \& Bowen, B. (1996). Targeting the child and family: A holistic approach to treating child and adolescent depressive disorders. In E. Hibbs \& P. Jensen (Eds.), Psychosocial treatments for child and adolescent disorders: Empirically based strategies for clinical practice (pp. 207-238). Washington, DC: American Psychological Association.

*Taylor, L., Adelman, H., \& Kaser-Boyd, N. (1986). The Origin Climate Questionnaire as a tool for studying psychotherapeutic process. Journal of Child and Adolescent Psychotherapy, 3, 10-16.

*Truax, C. B., Altman, H., Wright, L., \& Mitchell, K. M. (1973). Effects of therapeutic conditions in child therapy. Journal of Community Psychology, 1, 313-318.

*Van Orman, W. (1996). The relationship between therapeutic alliance and therapy outcome in home based family therapy. Unpublished doctoral dissertation, Boston University.

Weiss, B., \& Weisz, J. (1990). The impact of methodological factors on child psychotherapy outcome research: A meta-analysis for researchers. Journal of Abnormal Child Psychology, 18, 639-670.

Weisz, J., Donenberg, G., Han, S., \& Kauneckis, D. (1995). Child and adolescent psychotherapy outcomes in experiments versus clinics: Why the disparity? Journal of Abnormal Child Psychology, 23, 83-106.

Weisz, J., Huey, S., \& Weersing, V. R. (1998). Psychotherapy outcome research with children and adolescents. Advances in Clinical Child Psychology, 20, 49-91.

Weisz, J., Weiss, B., Alicke, M., \& Klotz, M. (1987). Effectiveness of psychotherapy with children and adolescents: A meta-analysis for clinicians. Journal of Consulting and Clinical Psychology, 55, 542-549.

Weisz, J., Weiss, B., Han, S., Granger, D., \& Morton, T. (1995). Effects of psychotherapy with children and adolescents revisited: A meta-analysis of treatment outcome studies. Psychological Bulletin, 117, 450-468.

Received December 13, 2001

Revision received March 29, 2002

Accepted May 6, 2002 